\title{
The effect of traditional music on mental status of chronic schizophrenic patients: a clinical trial study
}

\author{
Fatemeh Hakimi $^{\mathbb{(}}$, Elham Zarean ${ }^{2}$, Masoud Nikfarjam ${ }^{3 *}{ }^{(\mathbb{D}}$, Parastoo Yarmohammadi $^{(\mathbb{D}}$ \\ ${ }^{1} \mathrm{MD}$, Student Research Committee, Shahrekord University of Medical Sciences, Shahrekord, Iran. \\ ${ }^{2}$ Associate Professor, Department of Psychiatry, Islamic Research Committee, Shahrekord University of Medical Sciences, \\ Shahrekord, Iran. \\ ${ }^{3}$ Assistant Professor, Department of Psychiatry, School of Medicine, Shahrekord University of Medical Sciences, Shahrekord, \\ Iran. \\ ${ }^{4} \mathrm{PhD}$ Student, Social Determinants of Health Research Center, Department of Health Education and Health Promotion, School \\ of Public Health, Shahid Sadoughi University of Medical Sciences, Yazd, Iran.
}

*Corresponding Author: Masoud Nikfarjam, Department of Psychiatry, School of Medicine, Shahrekord University of Medical Sciences, Shahrekord, Tel: 09133838936, Email: nikmas140@gmail.com

\begin{abstract}
Background and aims: Music therapy is a simple and inexpensive method for the treatment of some psychiatric disorders. The aim of this study was to determine the effect of traditional music on the mental state of hospitalized chronic schizophrenia patients.

Methods: In this clinical trial, 30 schizophrenia patients in the intervention group received routine treatment and attended music therapy sessions and 30 patients in the control group received routine treatment alone. Group music therapy sessions with traditional music were conducted 5 times a week for 2 months. Demographic questionnaire and Andreasen's positive and negative symptoms questionnaires were completed at baseline and after the completion of music therapy sessions. Data analysis was conducted in SPSS version 16.0 using independent and paired $t$ tests.

Results: The mean scores of delusion $(P=0.001)$ and bizarre behavior $(P=0.036)$ and the total score of the SAPS $(P=0.001)$ significantly decreased after intervention in the music therapy group. However, in the control group, the differences in the total and individual domain scores of the SAPS were not significant $(P>0.05)$. There was no significant difference between the control and music therapy groups in the total and individual domain scores of the Scale for the Assessment of Negative Symptoms (SANS) before and after the intervention $(P>0.05)$. Additionally, before and after the study, the mean score of avolition in the music therapy group was significantly lower compared to the control group $(P=0.03)$. The mean score of alogia in the music therapy group was significantly lower compared to the control group $(P=0.01)$.

Conclusion: Music therapy is effective in improving most of the positive symptoms of schizophrenia and some of its negative aspects, and therefore can be used as a complementary therapy along with drug therapy.

Keywords: Schizophrenia, Positive symptoms, Negative symptoms, Music therapy
\end{abstract}

Received: 24 February 2020, Accepted: March 15 2020, ePublished: 29 June 2020

\section{Introduction}

Schizophrenia is a type of chronic psychological disorder that has various symptoms including hallucinations, delusions, cognitive dysfunction, and disturbed (catatonic) speech or behavior (1).

The development of schizophrenia is due to abnormalities of dopaminergic and serotonergic neurotransmitters, hypoactivity of GABA, and alphaadrenergic or glutaminergic hyperactivity. Genetics also plays a key role in the development of the disease (2). Schizophrenia is a disorder of the brain that is associated with psychotic symptoms, rather than a mental disorder. The disease has a negative impact on the quality of life of patients with neuropsychotic symptoms and disrupts all body functions (3). The early onset of schizophrenia, along with its chronic episodes, turns the disease into a debilitating disorder for many patients and their families, which leads to numerous mental, emotional, and social problems (4-6). Although the current treatments mainly consist of antipsychotics combined with psychological therapies, social support, and rehabilitation, there is a pressing need for more effective treatments and development of new and complementary therapies $(7,8)$. One of the non-pharmacological approaches to treating psychiatric disorders is the use of pleasant acoustic stimuli, known as music therapy $(9,10)$. Music therapy is a nonpharmacological and complementary therapy that improves the treatment and well-being of patients by increasing stress thresholds and eliminating negative emotions, regulating internal processes, maintaining relaxation, and

(C) 2020 The Author(s); Published by Shahrekord University of Medical Sciences. This is an open-access article distributed under the terms of the Creative Commons Attribution License (http://creativecommons.org/licenses/by/4.0), which permits unrestricted use, distribution, and reproduction in any medium, provided the original work is properly cited. 
enhancing immunity $(11,12)$, and it helps psychosocial, physiological, and emotional integration throughout the treatment of illness and disability (10). However, this issue needs further studies because psychiatric patients have different psychological conditions and may not have the same reactions in response to external acoustic stimuli (12). On the other hand, music therapy has no negative side effects and is relatively inexpensive (13). Therefore, the present study was conducted to determine the effect of Bakhtiari traditional music on the mental state of chronic schizophrenic patients admitted to Sina hospital.

\section{Materials and Methods}

This double-blind clinical trial was performed on schizophrenic patients hospitalized in Sina hospital (west of Iran) in 2015. The intervention group $(n=30)$ attended music therapy sessions and the control group $(n=30)$ received routine treatments for schizophrenia. Patients were selected by convenience sampling method and then randomly divided into two groups of intervention and control. The study was a double-blind study in which each patient was given a code, which indicated which group they belonged to, and the researcher did not know which patient enrolled in the control or treatment group. The sample size was determined to be 30 patients per group according to similar studies (13-15) based on the following formula with $95 \%$ confidence interval and $80 \%$ power.

$n=\frac{\left(z_{1}+z_{2}\right)^{2}\left(2 s^{2}\right)}{d^{2}} \cong 30$

$Z_{1-\alpha / 2}=1.96$

$Z_{1-\beta}=0.84$

$S$ : An estimate of the standard deviation of the variable was considered.

d: The accuracy was considered to be 0.7 of $S$.

The inclusion criteria were chronic schizophrenia, hospitalization, lack of mental retardation, hearing impairment and severe physical disabilities, ability to attend group sessions, and at least 18 years of age. The exclusion criteria were lack of volunteering to participate in the study and experience of psychotic distress or discomfort during the intervention.

Demographic information included age, sex, and duration of hospital stay. In addition, two questionnaires of positive and negative symptoms developed by Andreasen were used to evaluate the positive and negative symptoms of the patients. The Scale for the Assessment of Positive Symptoms (SAPS) consists of 33 items to investigate symptoms such as hallucinations, delusions, bizarre behaviors, and thought disorders. The choices include none to suspected (rated $0-1$ ), mild to moderate (rated 2-3), and severe to excessive (rated 4-5).

The higher the score, the more severe the disease. The Scale for the Assessment of Negative Symptoms (SANS) addresses certain symptoms such as affective blunting, poverty of speech, aimlessness, lack of affection, lack of pleasure and social apathy and inattention. The items $(n=24)$ are scaled similarly to those of the SAPS (16).

The construct validity and reliability of the positive and negative symptom questionnaires were assessed based on Iranian cultural context and adaptation was performed (17).

In a study conducted by Khalafbaigi et al, the coefficients of reliability for five domains of positive symptoms were calculated by internal consistency method and coefficients for all symptoms were significant. The coefficient of reliability was found to be 0.78 for the negative symptoms and 0.77 for the positive symptoms (18).

Demographic questionnaires, SAPS, and SANS were completed at the beginning of the study and after the completion of music therapyx sessions. In the intervention group, in addition to routine treatment, group music therapy was delivered in five sessions per week for two months.

In the control group, only routine treatment was delivered and during two months of intervention, patients did not use music and similar interventions. Music therapy sessions lasted 60 minutes in which Bakhtiari folk music was replayed while patients were active and danced. After the completion of the intervention, both positive and negative symptoms were assessed in both groups.

Data were analyzed by SPSS version 16.0 using descriptive statistics including the mean and inferential statistics including independent $t$ test. The KolmogorovSmirnov test was used to test the normal distribution of the data.

Results

In this study, 60 patients were divided into control and music therapy groups. There were 10 women and 20 men in each group. The mean age $(P=0.91)$ and mean length of stay $(P=0.17)$ were similar between the two groups and the groups were not significantly different (Table 1).

The Kolmogorov-Smirnov test showed that the distribution of data in this study was normal according to the total score of positive and negative symptoms and demographic variables $(P<0.05)$. At the baseline, independent $t$ test showed that the two groups were similar in total and individual domain scores of the SAPS and SANS and there was no statistically significant difference (Tables 2 and 3).

The comparison of total and individual domain scores of the SAPS before and after intervention in music therapy

Table 1. Comparison of age and duration of hospitalization in the two study groups

\begin{tabular}{lccc}
\hline \multirow{2}{*}{ Variable } & Control & Intervention & \\
\cline { 2 - 3 } & Mean \pm SD & Mean \pm SD & \\
\hline Age & $41.6 \pm 46.73$ & $41.9 \pm 23.05$ & 0.91 \\
Duration of hospitalization & $7.40 \pm 83.80$ & $9.30 \pm 26.17$ & 0.17 \\
\hline
\end{tabular}


Table 2. Comparison of the total and individual domain scores of the SAPS before and after intervention in control and music therapy groups

\begin{tabular}{|c|c|c|c|c|}
\hline \multirow{2}{*}{ Variables } & & \multirow{2}{*}{$\begin{array}{c}\text { Control } \\
\text { Mean } \pm S D\end{array}$} & \multirow{2}{*}{$\frac{\text { Intervention }}{\text { Mean } \pm \text { SD }}$} & \multirow{2}{*}{$P$ value } \\
\hline & & & & \\
\hline \multirow{2}{*}{ Hallucination } & Before & $14.43 \pm 4.09$ & $15.70 \pm 3.46$ & 0.20 \\
\hline & After & $14.40 \pm 2.83$ & $14.50 \pm 0.49$ & 0.84 \\
\hline \multicolumn{2}{|l|}{$P$ value } & 0.97 & 0.06 & \\
\hline \multicolumn{2}{|c|}{$\begin{array}{l}\text { Differences before and after the } \\
\text { intervention }\end{array}$} & $-0.03 \pm 1.54$ & $-1.20 \pm 2.75$ & 0.04 \\
\hline \multirow{2}{*}{ Delusion } & Before & $30.73 \pm 4.43$ & $30.90 \pm 4.48$ & 0.88 \\
\hline & After & $29.93 \pm 6.78$ & $26.20 \pm 3.56$ & $0.009^{*}$ \\
\hline \multicolumn{2}{|l|}{$P$ value } & 0.59 & $0.001^{*}$ & \\
\hline \multicolumn{2}{|c|}{$\begin{array}{l}\text { Differences before and after the } \\
\text { intervention }\end{array}$} & $-0.80 \pm 3.27$ & $-4.70 \pm 6.30$ & $0.004^{*}$ \\
\hline \multirow{2}{*}{ Bizarre behavior } & Before & $9.83 \pm 2.03$ & $10.50 \pm 3.39$ & 0.36 \\
\hline & After & $9.43 \pm 3.65$ & $9 \pm 1.80$ & 0.56 \\
\hline \multicolumn{2}{|l|}{$P$ value } & 0.60 & $0.03^{*}$ & \\
\hline \multicolumn{2}{|c|}{$\begin{array}{l}\text { Differences before and after the } \\
\text { intervention }\end{array}$} & $-0.40 \pm 1.67$ & $-1.50 \pm 2.67$ & 0.06 \\
\hline \multirow{2}{*}{$\begin{array}{l}\text { Formal thought } \\
\text { disorder }\end{array}$} & Before & $17.83 \pm 2.69$ & $19.36 \pm 4.68$ & 0.12 \\
\hline & After & $18.83 \pm 9.50$ & $17.10 \pm 6.50$ & 0.41 \\
\hline \multicolumn{2}{|l|}{$P$ value } & 0.58 & 0.12 & \\
\hline \multicolumn{2}{|c|}{$\begin{array}{l}\text { Differences before and after the } \\
\text { intervention }\end{array}$} & $1.00 \pm 3.61$ & $-2.26 \pm 4.70$ & $0.003^{*}$ \\
\hline \multirow{2}{*}{$\begin{array}{l}\text { Total score of positive } \\
\text { symptoms }\end{array}$} & Before & $72.83 \pm 8.06$ & $76.46 \pm 9.75$ & 0.12 \\
\hline & After & $72.60 \pm 14.80$ & $66.80 \pm 6.21$ & $0.049^{*}$ \\
\hline \multicolumn{2}{|l|}{$P$ value } & 0.94 & $0.001^{*}$ & \\
\hline \multicolumn{2}{|c|}{$\begin{array}{l}\text { Differences before and after the } \\
\text { intervention }\end{array}$} & $-0.23 \pm 5.37$ & $-9.66 \pm 13.18$ & $0.001^{*}$ \\
\hline
\end{tabular}

group based on paired $t$-test showed that the mean scores of delusion $(P=0.001)$ and bizarre behavior $(P=0.03)$ and total score of the SAPS $(P=0.001)$ significantly decreased after the intervention. However, in the control group, the difference in the total and individual domain scores of the SAPS before and after the intervention was not significant $(P>0.05)$. Moreover, based on the results of independent $t$-test, there was a significant difference between the two groups in terms of the mean scores of delusion $(P=0.004)$ and inferential thinking $(P=0.003)$ and total score of the SAPS $(P=0.001)$ before and after study. This difference was higher in the music therapy group compared to the control group and the mean scores of delusion and inferential thinking and the total score of SAPS were lower in the music therapy group compared to the control group.

Based on the results of the paired $t$-test, there was no significant difference between the control and music therapy groups before and after intervention in terms of total and individual domain scores of the SANS $(P>0.05)$. Moreover, based on the results of the independent $t$ test, there was a significant difference between the two groups before and after the intervention in terms of the mean score of avolition $(P=0.03)$. The mean score of avolition was lower in the music therapy group than in the control group. In addition, the mean difference between the control and music therapy groups in alogia was significant after the intervention, indicating that the mean score in the music therapy group was significantly lower compared to the control group $(P=0.01)$ (Table 3).

\section{Discussion}

The aim of this study was to determine the effect of traditional music on the mental status of chronic schizophrenic patients admitted to Sina hospital of Joneghan. In the present study, the mean scores of delusion and bizarre behavior and the total score of the SAPS significantly reduced after the intervention in the music therapy group, and the mean scores of delusion and formal thinking and total score of the SAPS were significantly lower in the music therapy group than in the control group. In addition, the mean score of avolition in the music therapy group was lower compared to the control group. In addition, the mean score of alogia was

Table 3. Comparison of total and individual domain scores of the SANS before and after intervention in control and music therapy groups

\begin{tabular}{|c|c|c|c|c|}
\hline \multirow{2}{*}{ Variables } & & \multirow{2}{*}{$\begin{array}{c}\text { Control } \\
\text { Mean } \pm \text { SD }\end{array}$} & \multirow{2}{*}{$\begin{array}{c}\text { Intervention } \\
\text { Mean } \pm \text { SD }\end{array}$} & \multirow{2}{*}{$P$ value } \\
\hline & & & & \\
\hline \multirow{2}{*}{ Affective blunting } & Before & $13.10 \pm 2.33$ & $13.16 \pm 3.19$ & 0.92 \\
\hline & After & $13.40 \pm 4.83$ & $14.42 \pm 5.40$ & 0.47 \\
\hline \multicolumn{2}{|l|}{$P$ value } & 0.76 & 0.24 & \\
\hline \multicolumn{2}{|c|}{$\begin{array}{l}\text { Differences before and after the } \\
\text { intervention }\end{array}$} & $0.30 \pm 1.93$ & $1.26 \pm 1.00$ & 0.018 \\
\hline \multirow{2}{*}{ Alogia } & Before & $12.16 \pm 3.90$ & $10.86 \pm 3.30$ & 0.16 \\
\hline & After & $11.80 \pm 2.63$ & $9.83 \pm 3.01$ & $0.01^{*}$ \\
\hline \multicolumn{2}{|l|}{$P$ value } & 0.67 & 0.21 & \\
\hline \multicolumn{2}{|c|}{$\begin{array}{l}\text { Differences before and after the } \\
\text { intervention }\end{array}$} & $-0.36 \pm 1.79$ & $-1.03 \pm 2.39$ & 0.22 \\
\hline \multirow{2}{*}{ Avolition-Apathy } & Before & $10.63 \pm 2.73$ & $11.16 \pm 2.46$ & 0.43 \\
\hline & After & $10.40 \pm 1.60$ & $9.80 \pm 3.21$ & 0.36 \\
\hline \multicolumn{2}{|l|}{$P$ value } & 0.69 & 0.07 & \\
\hline \multicolumn{2}{|c|}{$\begin{array}{l}\text { Differences before and after the } \\
\text { intervention }\end{array}$} & $-0.23 \pm 1.19$ & $-1.36 \pm 2.59$ & $0.03 *$ \\
\hline \multirow{2}{*}{$\begin{array}{l}\text { Anhedonia- } \\
\text { asociality }\end{array}$} & Before & $11.76 \pm 2.43$ & $10.66 \pm 3.30$ & 0.14 \\
\hline & After & $11.56 \pm 3.86$ & $9.86 \pm 4.93$ & 0.14 \\
\hline \multicolumn{2}{|l|}{$P$ value } & 0.81 & 0.46 & \\
\hline \multicolumn{2}{|c|}{$\begin{array}{l}\text { Differences before and after the } \\
\text { intervention }\end{array}$} & $-0.20 \pm 1.59$ & $-0.80 \pm 2.61$ & 0.28 \\
\hline \multirow{2}{*}{ Inattention } & Before & $9.06 \pm 2.25$ & $8.13 \pm 2.77$ & 0.15 \\
\hline & After & $8.83 \pm 2.78$ & $7.37 \pm 3.15$ & 0.06 \\
\hline \multicolumn{2}{|l|}{$P$ value } & 0.72 & 0.32 & \\
\hline \multicolumn{2}{|c|}{$\begin{array}{l}\text { Differences before and after the } \\
\text { intervention }\end{array}$} & $-0.23 \pm 1.32$ & $-0.76 \pm 2.04$ & 0.23 \\
\hline \multirow{2}{*}{$\begin{array}{l}\text { Total score of } \\
\text { negative symptoms }\end{array}$} & Before & $56.73 \pm 8.08$ & $54.00 \pm 7.67$ & 0.18 \\
\hline & After & $\begin{array}{c}56.47 \pm \\
16.95\end{array}$ & $\begin{array}{c}51.30 \pm \\
14.38\end{array}$ & 0.20 \\
\hline \multicolumn{2}{|l|}{$P$ value } & 0.94 & 0.36 & \\
\hline \multicolumn{2}{|c|}{$\begin{array}{l}\text { Differences before and after the } \\
\text { intervention }\end{array}$} & $-0.26 \pm 5.90$ & $-2.70 \pm 7.67$ & 0.17 \\
\hline
\end{tabular}


significantly lower in the music therapy group compared to the control group. Consistent with the results of this study, a study conducted by Khalafbaigi et al confirms these findings. Their findings showed that music therapy is effective in raising memory and attention scores (18). Yang et al also found that after three months of music therapy, negative symptoms reduced in the schizophrenic group but no significant change was observed in the control group (19). Lu et al have also reported that group music therapy is an economical and convenient method that can be used to relieve depression and positive and negative symptoms in patients with schizophrenia (20). In another study aimed at investigating the effect of relaxation and music therapy on psychological symptoms and depression in patients with schizophrenia, the results showed that in the intervention group, the mean scores of the psychological symptoms and depression reduced.

Relaxation exercises and music therapy were also effective in reducing the positive and negative psychological symptoms as well as depression in schizophrenic patients (21). The results of another study indicated that group music therapy combined with standard care was effective in controlling the dose of neuroleptic drugs in patients with psychotic disorders such as schizophrenia (22).

A study conducted by Peng et al showed that 10 sessions of group music activity including singing and listening to music reduced the scores in total and subscales of the BPRS in patients with acute schizophrenia (13).

In addition, in their study, group music activity included both active and passive forms of music therapy. Group activities may have increased social interactions among patients, which increased the positive effects of these activities (13). In this study, it can be concluded that positive changes in patients' conditions were due to music therapy. The goal of music therapy is to enable the patient to develop relationships and express relationships and points of view that cannot be expressed through words by playing music and singing (23).

Music therapy can help people with schizophrenia improve mental state (general symptoms, depression, and anxiety), general and social functioning, global state, and quality of life in the short to medium term. Music therapy particularly seems to affect patients' motivational, emotional, and relational aspects and helps patients to improve their social activities and associated roles (24). Music therapy incorporates music experiences to help people with serious mental disorders develop relationships as they may not be able to use words alone to solve problems.

Previous studies have examined the effects of music therapy as a complementary therapy to standard treatments. However, the effects of music therapy appear to depend on the number of music therapy sessions (24). Therefore, further studies should be conducted with the control of confounding factors, and also to investigate longer-term symptoms.

\section{Conclusion}

Music therapy alone has an effect on the overall improvement of positive symptoms as well as on aspects of delusions, bizarre behavior, and formal thinking. Regarding negative symptoms, however, music is more effective in improving the subscales of avolition and alogia than other negative symptoms. In other words, music improves the patient's behavior, makes it more rational, and also improves his/her relationship with the environment. Based on these results, music can be used as a complementary therapy along with drug therapy.

Conflict of Interests

The authors declare that there is no conflict of interests.

Ethical Approval

This study protocol was approved by Shahrekord University of Medical Sciences (IR.SKUMS.REC.91-7-20) and it was also registered at the Iranian Clinical Trial Registry (identifier: IRCT201412282085N14).

Authors Contribution

$\mathrm{MN}$ and $\mathrm{FH}$ conceived and design the study, PY acquired data, $\mathrm{EZ}$ analyed and interpreted of data, $\mathrm{MN}$ and $\mathrm{FH}$ drafted of the manuscript, $\mathrm{MN}, \mathrm{FH}$ and $\mathrm{PY}$ did critical revision of the manuscript for important intellectual content, MN, FH, EZ and PY supervised and approved final draft.

\section{Funding/Support}

This study was financially supported by Shahrekord University of Medical Sciences.

Acknowledgments

This article was derived from a research project approved by the Research and Technology Deputy of the Shahrekord University of Medical Sciences (Approval No. 1798). Hereby, the researchers gratefully thank the patients who participated in this study.

\section{References}

1. Patel KR, Cherian J, Gohil K, Atkinson D. Schizophrenia: overview and treatment options. P T. 2014;39(9):638-45.

2. Hany M, Rehman B, Azhar Y, Chapman J. Schizophrenia. In: StatPearls. Treasure Island, FL: StatPearls Publishing; 2020. [Updated 2019 Dec 16]. Available from: https://www.ncbi. nlm.nih.gov/books/NBK539864/.

3. Kirkpatrick B, Miller B, García-Rizo C, Fernandez-Egea E. Schizophrenia: a systemic disorder. Clin Schizophr Relat Psychoses. 2014;8(2):73-9. doi: 10.3371/csrp.kimi.031513.

4. Lippi G. Schizophrenia in a member of the family: burden, expressed emotion and addressing the needs of the whole family. S Afr J Psychiatr. 2016;22(1):922. doi: 10.4102/ sajpsychiatry.v22i1.922.

5. Caqueo-Urízar A, Rus-Calafell M, Craig TK, Irarrazaval M, Urzúa A, Boyer $L$, et al. Schizophrenia: impact on family dynamics. Curr Psychiatry Rep. 2017;19(1):2. doi: 10.1007/ s11920-017-0756-z.

6. Mottaghipour $\mathrm{Y}$, Tabatabaee M. Family and patient psychoeducation for severe mental disorder in Iran: a review. Iran J Psychiatry. 2019;14(1):84-108.

7. Owen MJ, Sawa A, Mortensen PB. Schizophrenia. Lancet. 2016;388(10039):86-97. doi: 10.1016/s0140-6736(15)01121- 
8. Stępnicki P, Kondej M, Kaczor AA. Current concepts and treatments of schizophrenia. Molecules. 2018;23(8). doi: 10.3390/molecules23082087.

9. Hegde S. Music therapy for mental disorder and mental health: the untapped potential of Indian classical music. BJPsych Int. 2017;14(2):31-3. doi: 10.1192/s2056474000001732.

10. Wang $S$, Agius $M$. The use of music therapy in the treatment of mental illness and the enhancement of societal wellbeing. Psychiatr Danub. 2018;30(Suppl 7):595-600.

11. Stanczyk MM. Music therapy in supportive cancer care. Rep Pract Oncol Radiother. 2011;16(5):170-2. doi: 10.1016/j. rpor.2011.04.005.

12. Witusik A, Pietras T. Music therapy as a complementary form of therapy for mental disorders. Pol Merkur Lekarski. 2019;47(282):240-3.

13. Peng SM, Koo M, Kuo JC. Effect of group music activity as an adjunctive therapy on psychotic symptoms in patients with acute schizophrenia. Arch Psychiatr Nurs. 2010;24(6):429-34. doi: 10.1016/j.apnu.2010.04.001.

14. Chang SC, Chen CH. Effects of music therapy on women's physiologic measures, anxiety, and satisfaction during cesarean delivery. Res Nurs Health. 2005;28(6):453-61. doi: 10.1002/nur.20102.

15. Bekiroğlu T, Ovayolu N, Ergün Y, Ekerbiçer H. Effect of Turkish classical music on blood pressure: a randomized controlled trial in hypertensive elderly patients. Complement Ther Med. 2013;21(3):147-54. doi: 10.1016/j.ctim.2013.03.005.

16. Andreasen NC, Arndt S, Miller D, Flaum M, Nopoulos P. Correlational studies of the Scale for the Assessment of Negative Symptoms and the Scale for the Assessment of Positive Symptoms: an overview and update. Psychopathology. 1995;28(1):7-17. doi: 10.1159/000284894.
17. Foruzandeh $N$, Foruzandeh $M$, Delaram $M$, Darakhshandeh S, Deris F, Hasanpour Dehkordi A, et al. The effect of occupational therapy on some aspects of quality of life in schizophrenic patients. Journal of Shahrekord University of Medical Sciences. 2009;10(4):51-7. [Persian].

18. Khalafbaigi $M$, Bayanzadeh A, Zadeh-mohammadi A, Shafaroodi $\mathrm{N}$. The effect of musical activity on memory and attention in schizophrenia. Iranian Journal of Psychiatry and Clinical Psychology. 2006;12(3):236-43. [Persian].

19. Yang WY, Li Z, Weng YZ, Zhang HY, Ma B. Psychosocial rehabilitation effects of music therapy in chronic schizophrenia. Hong Kong Journal of Psychiatry. 1998;8(1):38-40.

20. Lu SF, Lo CH, Sung HC, Hsieh TC, Yu SC, Chang SC. Effects of group music intervention on psychiatric symptoms and depression in patient with schizophrenia. Complement Ther Med. 2013;21(6):682-8. doi: 10.1016/j.ctim.2013.09.002.

21. Kavak F, Ünal S, Yılmaz E. Effects of relaxation exercises and music therapy on the psychological symptoms and depression levels of patients with schizophrenia. Arch Psychiatr Nurs. 2016;30(5):508-12. doi: 10.1016/j.apnu.2016.05.003.

22. Degli Stefani M, Biasutti M. Effects of music therapy on drug therapy of adult psychiatric outpatients: a pilot randomized controlled study. Front Psychol. 2016;7:1518. doi: 10.3389/ fpsyg.2016.01518.

23. Rafieyan R, Ries R. A description of the use of music therapy in consultation-liaison psychiatry. Psychiatry (Edgmont). 2007;4(1):47-52.

24. Geretsegger M, Mössler KA, Bieleninik $\measuredangle$, Chen XJ, Heldal TO, Gold C. Music therapy for people with schizophrenia and schizophrenia-like disorders. Cochrane Database Syst Rev. 2017;5(5):CD004025. doi: 10.1002/14651858.CD004025. pub4. 